\title{
Inhibidor de incrustaciones natural a base del mucílago de la hoja de cayena (Hibiscus rosa sinensis)
}

\author{
(Natural scale inhibitor based on mucilage \\ of the cayenne leaf (Hibiscus rosa sinensis)
}

\author{
Anabel Sarahis Reyes Sisco ${ }^{1}$, José Antonio Abraham Ruiz Arenas ${ }^{1}$, Luis Antonio Castillo Campos ${ }^{1}$
}

\begin{abstract}
Resumen
Las incrustaciones minerales representan uno de los problemas más frecuentes en la industria petrolera, ya que deterioran los sistemas de producción, ocasionando pérdidas de presión y, consecuentemente, la disminución de la producción de hidrocarburos. Con base en esta problemática, se realizó esta investigación cuyo propósito fue evaluar la eficiencia de un inhibidor de incrustaciones natural a base del mucílago de la hoja de la planta de cayena (Hibiscus rosa sinensis) en muestras de agua sintética y de producción. Para ello, se realizó la caracterización físico-química de las muestras de agua, así como del mucílago previamente extraído de la planta. Posteriormente, se formuló y caracterizó el inhibidor a tres concentraciones diferentes $(2,4$ y $6 \% \mathrm{~m} / \mathrm{V})$ y se evaluó la eficiencia del producto en ambas muestras de agua, considerando tres (3) dosis diferentes (55, 100 y 500 ppm) y siguiendo los lineamientos planteados en la norma NACE TM0347-2007, resultando más eficiente el inhibidor de $2 \%$ m/V en su dosis más alta. Adicionalmente, se utilizó un inhibidor comercial de la empresa Halliburton para fines comparativos en un análisis estadístico que indicó la ausencia de una diferencia estadísticamente significativa entre el producto a $2 \% \mathrm{~m} / \mathrm{V}$ y el inhibidor comercial.
\end{abstract}

\section{Palabras clave}

Mucílago; cayena; inhibidor; incrustaciones; agua de producción.

\begin{abstract}
Nowadays, mineral incrustations are one of the most frequent problems in the petroleum industry; they deteriorate the production systems, causing pressure losses and, consequently, the decrease of hydrocarbon production. Based on this problem, this research was conducted with the purpose of evaluate the efficiency of a natural scale inhibitor based on the leaf mucilage of the cayenne plant (Hibiscus rosa sinensis) in synthetic and production water samples. In order to do this, the physical-chemical characterization on the water samples was carried out, as well as the mucilage previously extracted from the plant. Subsequently, the natural inhibitor was formulated and characterized at three different concentrations $(2,4$ and $6 \% \mathrm{~m} / \mathrm{V})$ and the efficiency of the product was evaluated in both water samples, considering three (3) different doses (55, 100 and 500 ppm) following the guidelines of the NACE standard TM0347-2007, with the result that the inhibitor of $2 \% \mathrm{~m} / \mathrm{V}$ was the most efficient at its highest dose. In addition, the commercial inhibitor from Halliburton Company was used for comparative purposes in a statistical analysis that indicated the absence of a significant statistical difference between the product at $2 \% \mathrm{~m} / \mathrm{V}$ and the commercial inhibitor.
\end{abstract}

\section{Keywords}

Mucilage; cayenne; inhibitor; scales; production water.

\section{Introducción}

El petróleo a lo largo de los años se ha convertido en un recurso fundamental para el desarrollo de las naciones; sin embargo, al encontrarse bajo miles de metros de profundidad, su extracción no resulta una tarea fácil. Es conocido que el llamado "oro negro" suele producirse acompañado de gas y de agua, viéndose incrementada la producción de esta última a medida que transcurre el tiempo (Kelland, 2014). 
El agua que sale de la formación generalmente contiene grandes cantidades de sales minerales que bajo condiciones como flujos bajos o poco turbulentos, valores de $\mathrm{pH}$ elevados, tiempos de exposición prolongados y cambios de presión y temperatura, tienden a unirse dando lugar a la formación y posterior precipitación de compuestos cristalizados denominados incrustaciones (Canul y Cortés, 2015), las cuales se consideran como uno de los problemas que más preocupan a los ingenieros de producción durante los procesos de explotación de petróleo debido a que estos cristales, comúnmente de carbonato de calcio y magnesio, se adhieren a las superficies de los conductos, tuberías y accesorios, ocasionando la reducción del área de flujo, lo que a su vez conlleva a un aumento en la caída de presión y, en consecuencia, la disminución de la producción (Espinosa, 2015).

Las reparaciones de los daños causados por las incrustaciones por lo general resultan más complejas y costosas que la prevención de los mismos, por esta razón existen diversos tratamientos químicos, físicos y mixtos diseñados para minimizarlos, los cuales de acuerdo al mecanismo de acción que empleen permitirán evitar la nucleación y/o el crecimiento de cristales, dispersar los cristales ya formados evitando que se precipiten o actuando como agentes secuestrantes de los iones metálicos impidiendo que reaccionen químicamente (García, 2010).

Entre los inhibidores más usados destacan los de base sintética ya que resultan altamente eficientes; no obstante, cuentan con sustancias químicas que no son amigables con el medio ambiente (Fevang, 2017). Por esta razón, en la actualidad se han venido desarrollando aditivos antiicrustantes orgánicos muy efectivos que tienen altas tasas de degradación y un bajo costo asociado. Destacando entre ellos, a nivel internacional los desarrollados por Guo, et al (2012), denominado "Preparación, caracterización y comportamiento de un inhibidor de incrustaciones a base de un copolímero modificado con quitosan", quienes a través de un método experimental estático (similar al empleado en el presente estudio), determinaron las influencias que tienen la concentración del copolímero, el tiempo de exposición a variaciones de temperatura, la concentración de $\mathrm{Ca}^{2+}$ y la concentración de $\mathrm{HCO} 3$ del sistema sobre la eficiencia de inhibición. De igual manera y, en el mismo año (2012), Chauhan et al, emplearon polímeros con base a pectina modificada como antiincrustante ecológico para la inhibición de incrustaciones de sulfato de calcio, para lo cual plantearon sintetizar copolímeros de injerto hidrolizados a base de pectina, investigando el efecto de los mismos en la formación e impedimento de precipitación de los cristales minerales.

En el ámbito nacional venezolano resalta el inhibidor de incrustaciones a base de Aloe Vera, patentado por INTEVEP, desarrollado por Viloria, Castillo, García y Biomorgi, en el año 2010 descrito como "un producto que comprende polisacáridos, solubilizados en agua entre $60{ }^{\circ} \mathrm{C}$ y $90^{\circ} \mathrm{C}$, además de la presencia de grupos funcionales de carboxilo y alcohol en las cadenas que interactúan con iones divalentes tales como $\mathrm{Ca}^{2+}$ y Mg${ }^{2+"}$ (Fink, 2010, 263). Además de los inhibidores de incrustación desarrollados en la Universidad de Oriente, Núcleo de Monagas, realizados por Rendón y Azocar (2016) y Fuenmayor y Peña (2016), quienes formularon productos inhibidores con base en pectina de Passiflora edulis, en presentación deshidratada y gel, respectivamente, empleando en ambos estudios, muestras de agua sintética a base de sulfato de calcio, y tomando en consideración la Norma NACE TM0374-2007, que se ajusta a una prueba de jarras en condición estática, tal cual como también se realizó en el presente estudio experimental.

Basándose en lo anterior, la presente investigación buscó desarrollar y evaluar un inhibidor de incrustaciones natural elaborado a partir de un mucílago obtenido de la hoja de la planta de cayena (Hibiscus rosa sinensis), con la finalidad de encontrar un producto innovador de origen nacional que pueda mitigar, o en el mejor de los casos solventar, la problemática ocasionada por la formación de incrustaciones, con un impacto mínimo en el ambiente y que además 
brinde los mismos beneficios de los inhibidores convencionales. Para ello se tomó como premisa o consideración hipotética que el producto desarrollado a distintas concentraciones permitiría mitigar a nivel experimental, la precipitación de incrustaciones de carbonato de calcio, tanto en muestras de agua sintética como de producción (proveniente del Distrito Morichal, al sur del estado Monagas, Venezuela). Para corroborar esto, fueron caracterizadas dichas muestras de aguas, así como el mucílago de la hoja de la planta de Hibiscus rosa sinensis y los productos anti incrustantes formulados, todo ello a través de distintas propiedades fisicoquímicas propias de cada fluido en cuestión. A continuación se procedió a evaluar la eficiencia del producto propuesto (a diferentes concentraciones), tomando como norma, la NACE TM0374-2007, y finalmente fueron comparadas las eficiencias obtenidas del agente químico experimental con las de un inhibidor comercial, a través de un análisis de varianza (ANOVA), con el propósito de establecer si existían diferencias estadísticas significativas entre ambos.

\section{Metodología}

\subsection{Caracterización de las muestras de agua}

Inicialmente se realizó la formulación de un agua sintética cuya composición fue de $6.659 \mathrm{~g} / \mathrm{l}$ de cloruro de calcio $\left(\mathrm{CaCl}_{2}\right)$ y $5.04 \mathrm{~g} / \mathrm{l}$ de bicarbonato de sodio $\left(\mathrm{NaHCO}_{3}\right)$, esta fue caracterizada en conjunto con una muestra de agua de producción obtenida de las estaciones de flujo PM-1 y PM-2 pertenecientes a la unidad de pesado del Distrito Morichal en el estado Monagas; todo ello a través de pruebas fisicoquímicas llevadas a cabo en los Laboratorios de Perforación, Yacimientos y Procesamiento de Hidrocarburos del Núcleo de Monagas de la Universidad de Oriente, Campus Los Guaritos. Entre las pruebas realizadas se tienen:

- Densidad: determinada según lo estipulado en la norma ASTM D854.

- Contenido de sólidos: obteniéndose tanto la cantidad de sólidos totales y suspendidos, siguiendo la norma COVENIN 2461-87, como el porcentaje de sólidos disueltos, según lo pautado en la práctica COVENIN 2342-86.

- $\quad$ pH: determinado considerando la norma COVENIN 2462-87.

- Alcalinidad: siguiendo los lineamientos de la práctica API RP 13B-1.

- Cloruros: a través de los planteamientos de la norma COVENIN 3141-1995.

- Dureza cálcica: cumpliendo con lo establecido en la norma COVENIN 2771-91.

- Índice de saturación de Langelier (LSI): calculado mediante las siguientes expresiones, donde: pHa: pH actual del agua; pHs: pH de saturación; TDS: total de sólidos disueltos; $T^{\circ} \mathrm{C}$ : temperatura del agua en grados centígrados.

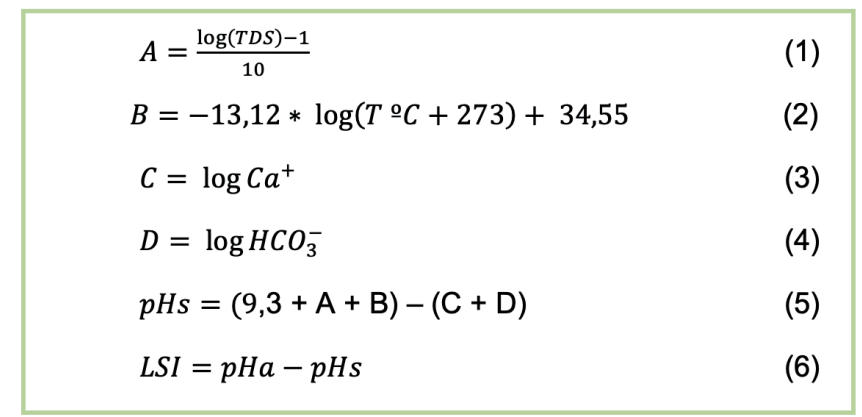




\subsection{Obtención del mucílago}

Posteriormente se realizó la extracción y caracterización del mucílago, para ello se utilizaron hojas de cayena, las cuales se recogieron directamente de la planta, se lavaron y secaron bajo la sombra durante 24 horas, adicionalmente, se procedió a secarlas en un horno a una temperatura entre 30 y $40{ }^{\circ} \mathrm{C}$ y, finalmente, su tamaño fue reducido con la ayuda de una trituradora. Seguidamente, la obtención del mucílago se realizó en dos pasos:

Paso n. ${ }^{0}$ 1: Extracción del mucílago: las hojas en polvo se colocaron en un vaso precipitado de $1000 \mathrm{ml}$ con $500 \mathrm{ml}$ de agua destilada durante al menos 3-4 horas con agitación continua a $60^{\circ} \mathrm{C}$. Luego, la solución concentrada se filtró a través de tela de muselina con el fin de separar las hojas del filtrado, el cual se refrigeró a una temperatura entre 3 y $4^{\circ} \mathrm{C}$.

Paso n. ${ }^{0}$ 2: Aislamiento de mucílago: se añadió alcohol absoluto, la cantidad de tres veces el volumen de filtrado para que se produjera la precipitación del mucílago, mismo que se recogió por medio de filtración por tela de muselina. Adicionalmente, el mucílago se secó en un horno a una temperatura inferior a $40^{\circ} \mathrm{C}$, seguidamente se pulverizó y almacenó en un recipiente hermético.

Para la caracterización se determinaron la densidad y pH del mucílago obtenido, siguiendo las normas mencionadas anteriormente, además de las pruebas descritas a continuación:

- Color y apariencia: mediante descripción visual.

- Viscosidad: se estimó a una temperatura externa de $25^{\circ} \mathrm{C}$ empleando un viscosímetro Brookfield, según lo especificado en la norma ASTM D2196.

- Rendimiento: se determinó con el uso de balanzas analíticas por medio de la siguiente expresión:

$$
\% R=\frac{\text { masa mucílago obtenido }}{\text { masa hojas molidas }} \times 100
$$

- Solubilidad: se calculó mediante la relación entre el mucílago no soluble y el mucílago total diluido en $100 \mathrm{ml}$ de agua destilada, tal y como lo establece la norma ASTM E1148-02(2008).

- Tamaño de partículas: se determinó mediante un análisis granulométrico efectuado en el Laboratorio de Suelos de la Universidad de Oriente, Núcleo de Monagas, Campus Juanico; tomando en consideración la norma ASTM C-136-01 y empleando tamices de diferentes tamaños.

- \% de humedad: se obtuvo relacionando la pérdida de peso del mucílago entre el peso de este sin secar.

- Estabilidad térmica: se empleó el método desarrollado por Malavé (2016), fijando tres temperaturas, a las cuales fue sometida cierta cantidad de mucílago contenida en un vial de masa conocida cerrado herméticamente.

- Identificación de grupos funcionales: a través de espectroscopía infrarroja realizada con un espectrómetro FT-IR (Nicolet is10), ubicado en el Laboratorio de Tamices Moleculares de la Universidad de Oriente, Núcleo de Monagas, Campus Juanico, y siguiendo lo estipulado en las normas ASTM E168 y ASTM E1252. 


\subsection{Desarrollo del inhibidor}

Para el desarrollo del inhibidor de incrustaciones, se utilizaron tres (3) concentraciones diferentes: 2 , 4 y $6 \% \mathrm{~m} / \mathrm{V}$ del mucílago previamente obtenido, en conjunto con tres dosis especificadas por la norma NACE TM 0374-2007 (55, 100 y 500 ppm) para la generación de $100 \mathrm{ml}$ de producto. Además, se agregaron $0.20 \mathrm{~g}$ de ácido cítrico a cada muestra con el propósito de retardar la descomposición del mucílago. Posteriormente, se caracterizó el producto formulado en conjunto con un inhibidor comercial mediante pruebas fisicoquímicas que permitieron determinar para ambos casos parámetros como: $\mathrm{pH}$, densidad, viscosidad, cloruros y dureza cálcica. Adicionalmente, para el inhibidor formulado se determinó contenido de sólidos totales, disueltos y en suspensión, siguiendo todos los procedimientos establecidos previamente.

Finalmente, para la evaluación del producto elaborado se siguieron los parámetros establecidos en la Norma Internacional NACE TM 0374, la cual establece que las pruebas para medir la capacidad que tiene el producto de evitar la precipitación de carbonato de calcio $\left(\mathrm{CaCO}_{3}\right)$ se llevan a cabo en condición estática, a presión atmosférica y con una duración de 24 horas. Para esto se realizaron pruebas de precipitación de carbonato de calcio a diversas concentraciones de inhibidor. El cálculo de porcentaje de inhibición se realizó mediante la siguiente expresión:

$$
\text { \%Inhibición }=\frac{\mathrm{Ca}-\mathrm{Cb}}{\mathrm{Cc}-\mathrm{Cb}} \times 100
$$

Donde:

Ca: dureza cálcica en ppm de la muestra tratada después de la precipitación; Cb: dureza cálcica en ppm del blanco después de la precipitación (blanco caliente); Cc: dureza cálcica en ppm del blanco antes de la precipitación (blanco frío).

Una vez obtenidos los resultados fueron sometidos a un procedimiento ANOVA o Análisis de Varianza, realizado mediante la utilización del programa Statgraphics Centurion XVI, con el objetivo de comparar y determinar si existen variaciones estadísticamente significativas entre los diferentes comportamientos.

\section{Resultados y discusión}

Las dos muestras de agua evaluadas se caracterizaron por presentar un elevado poder incrustante ya que se considera que el mismo se verá incrementado mientras mayor sea la dureza cálcica debido a que los compuestos carbonatados se transforman en cristales del elemento correspondiente por la elevación de temperatura, creando un material duro y resistente que se almacena en las paredes internas de las tuberías y accesorios (Fevang, 2017; Castillo, 2016), además, los valores obtenidos pueden considerarse bastante altos, posicionando las muestras como aguas "muy duras" al superar las 300 ppm establecidas en la clasificación COVENIN 277191. Esta tendencia incrustante se corroboró con los resultados arrojados para el resto de las propiedades determinadas y con el valor obtenido para el Îndice de Saturación de Langelier (LSI) que resultó ser mayor a cero en ambos casos, tal y como se observa en la tabla 1. 
Tabla 1. Propiedades de las muestras de agua

\begin{tabular}{|l|c|c|c|}
\hline \multicolumn{1}{|c|}{ Propiedad } & Unidad & Agua sintética & Agua de producción \\
\hline $\mathrm{pH}$ & - & 6.70 & 7.10 \\
\hline alcalinidad parcial & $\mathrm{ppm}$ & 0 & 0 \\
\hline alcalinidad total & $\mathrm{ppm}$ & 1.400 & 500 \\
\hline densidad & $\mathrm{g} / \mathrm{mL}$ & 1.0018 & 1.0128 \\
\hline sólidos totales & $\mathrm{ppm}$ & 10770 & 34332 \\
\hline cloruros & $\mathrm{ppm}$ & 499.89 & 20995.27 \\
\hline dureza cálcica & $\mathrm{ppm}$ & 1232 & 1680 \\
\hline índice de saturación de angelier & - & 0.8597 & 0.8846 \\
\hline
\end{tabular}

El mucílago a emplearse como materia prima presentó una coloración gris verdosa y una apariencia sólida pero maleable con una consistencia gelatinosa en su estado húmedo (Figura 1), sin embargo, una vez deshidratado adquirió una tonalidad verde oscuro la cual se aclaró un poco después de molido el producto, resultando un polvo color verde (Figura 2).

Figura 1. Mucílago húmedo

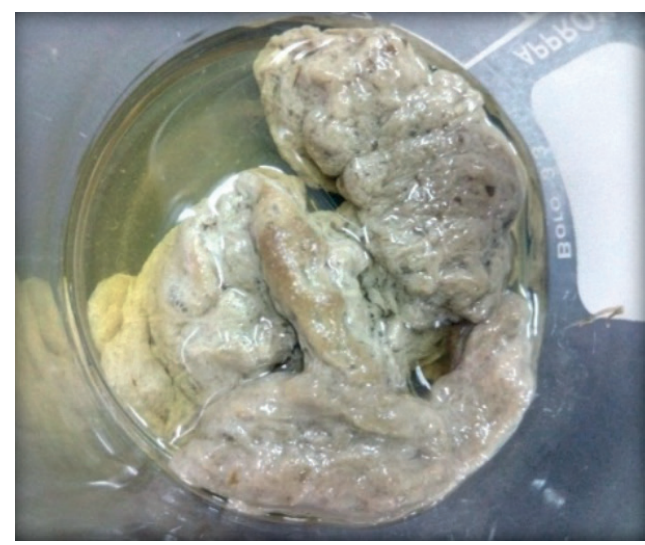

Figura 2. Mucílago deshidratado

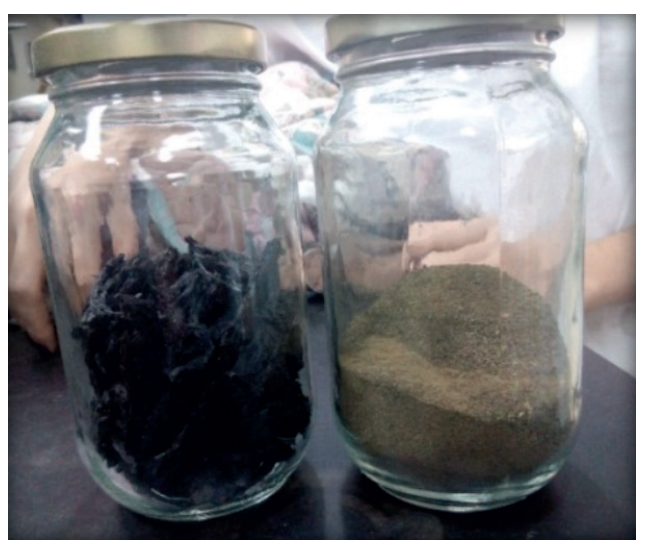

Dicho polvo, realizado el análisis granulométrico, presentó diferentes tamaños de partículas, predominando las de 150 y 250 micrones, tal y como se observa en la Figura 3.

Figura 3. Análisis granulométrico del mucílago extraído de la hoja de cayena (Hibiscus rosa sinensis)

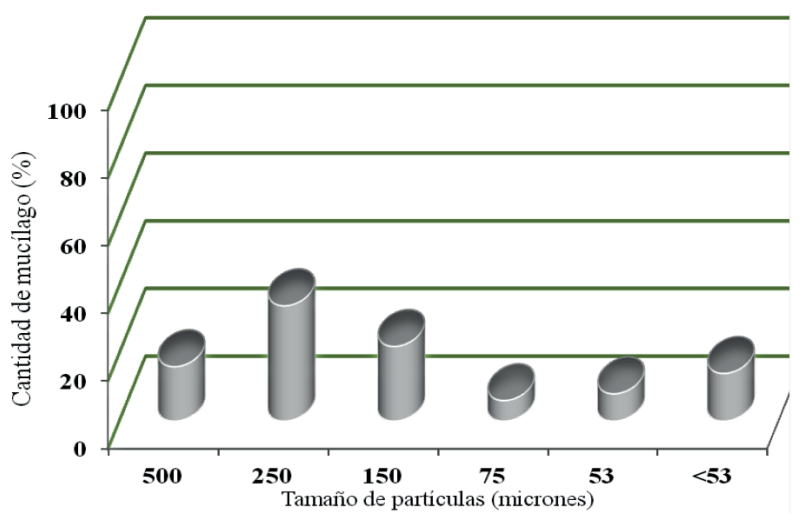

Para determinar las propiedades que requerían de una muestra líquida se realizó una dilución del polvo en agua destilada a una concentración 1\%m/V. Con la misma y, tal como se aprecia 
en la Tabla 2, gracias a las pruebas químicas se determinó la existencia de iones cloro y calcio en la composición del polisacárido; también se calculó el pH que resultó igual a 6.10, clasificándolo como una sustancia ácida. De igual forma, la densidad obtenida fue $0.9981 \mathrm{~g} / \mathrm{ml}$, al igual que la gravedad específica; el hecho que ambos valores sean menores a la densidad del agua común (1 $\mathrm{g} / \mathrm{ml}$ ) le confirió a las partículas del mucílago cierta facilidad para solubilizarse en ella, lo cual se reflejó en el 77.60 \% de solubilidad calculado para el material, ratificando que se trata de una sustancia vegetal hidrófila con gran afinidad por el agua y que, por ende, presentó además un 72.70 $\%$ de humedad. Esta capacidad de absorción se vio reflejada en el rendimiento obtenido para el mucílago, siendo de $11.56 \%$ en húmedo y de $3.07 \%$ deshidratado; porcentajes que se consideran dentro del rango habitual para este tipo de polisacáridos si se compara con el $9.12 \%$ obtenido por Luzuriaga (2012) para un mucílago de cacao, pero presentando un rendimiento de extracción medianamente bueno si se compara con el de otras plantas como la Opuntia cochenillifera (L.) Miller, la cual posee valores alrededor de 20 \% a 30 \% (Monrroy, et al., 2017). Análogo a esto, métodos utilizados para la extracción del mucílago de Nopal indican rendimientos muy bajos en comparación con los obtenidos para la planta de cayena: 0.3-0.84 \% (Mendoza, et al., 2014)

Tabla 2. Propiedades del mucílago extraído de la hoja de cayena (Hibiscus rosa sinensis)

\begin{tabular}{|l|l|l|}
\hline \multicolumn{1}{|c|}{ Propiedad } & \multicolumn{1}{c|}{ Unidad } & \multicolumn{1}{c|}{ Resultado } \\
\hline color y apariencia & - & verde \\
\hline densidad & $\mathrm{g} / \mathrm{mL}$ & 0.9981 \\
\hline gravedad específica & - & 0.9981 \\
\hline $\mathrm{pH}$ & - & 6.10 \\
\hline viscosidad & $\mathrm{cPs}$ & 12.06 \\
\hline rendimiento (húmedo) & $\%$ & 1.56 \\
\hline rendimiento (deshidratado) & $\%$ & 3.07 \\
\hline solubilidad & $\%$ & 77.60 \\
\hline humedad & $\%$ & 72.70 \\
\hline dureza cálcica & $\mathrm{ppm}$ & 328 \\
\hline cloruros & $\mathrm{ppm}$ & 9.9977 \\
\hline
\end{tabular}

Térmicamente, el mucílago presentó resistencia hasta temperaturas de alrededor de los $70^{\circ} \mathrm{C}$, a partir de la cual comenzó a hacerse inestable, alcanzando un estado crítico de degradación a temperaturas superiores a los $125^{\circ} \mathrm{C}$ y largos tiempos de exposición, lo cual se ve reflejado en la Figura 4.

Figura 4. Estabilidad térmica del mucílago de la hoja de cayena (Hibiscus rosa sinensis)

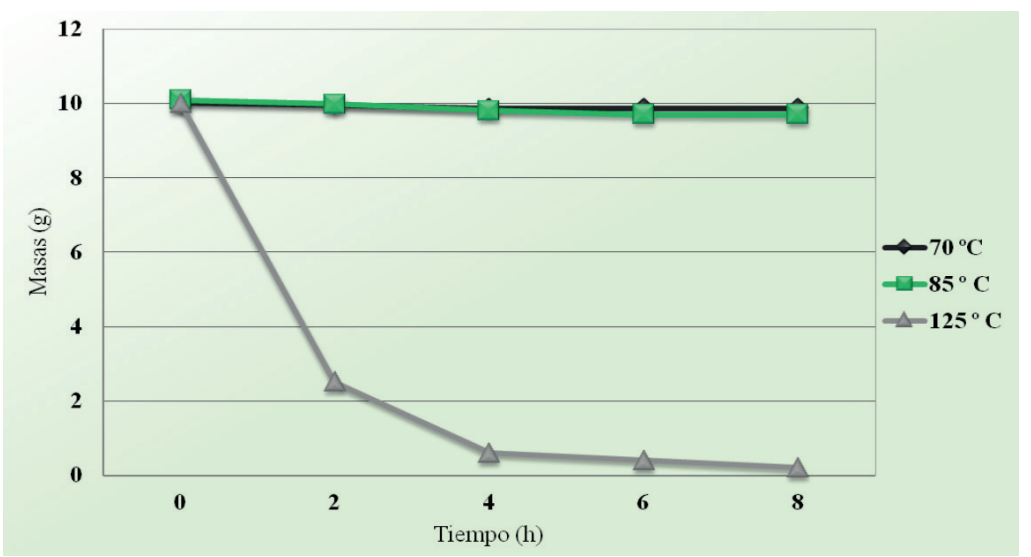


Por su parte, las variaciones en las curvas de absorbancia obtenidas en la prueba de espectroscopía infrarroja indicaron la presencia de grupos $\mathrm{OH}^{-}$(entre $3600-3100 \mathrm{~cm}^{-1}$ ), interacciones carbono - hidrógeno (3 000-2 $\left.900 \mathrm{~cm}^{-1}\right)$ grupos carbonilos $\left(1700-1500 \mathrm{~cm}^{-1}\right)$ y carboxílicos (1500 y $1200 \mathrm{~cm}^{-1}$ ) en la estructura del polisacárido (Figura 5).

Figura 5. Espectroscopía infrarroja del mucílago de la hoja de cayena (Hibiscus rosa sinensis)

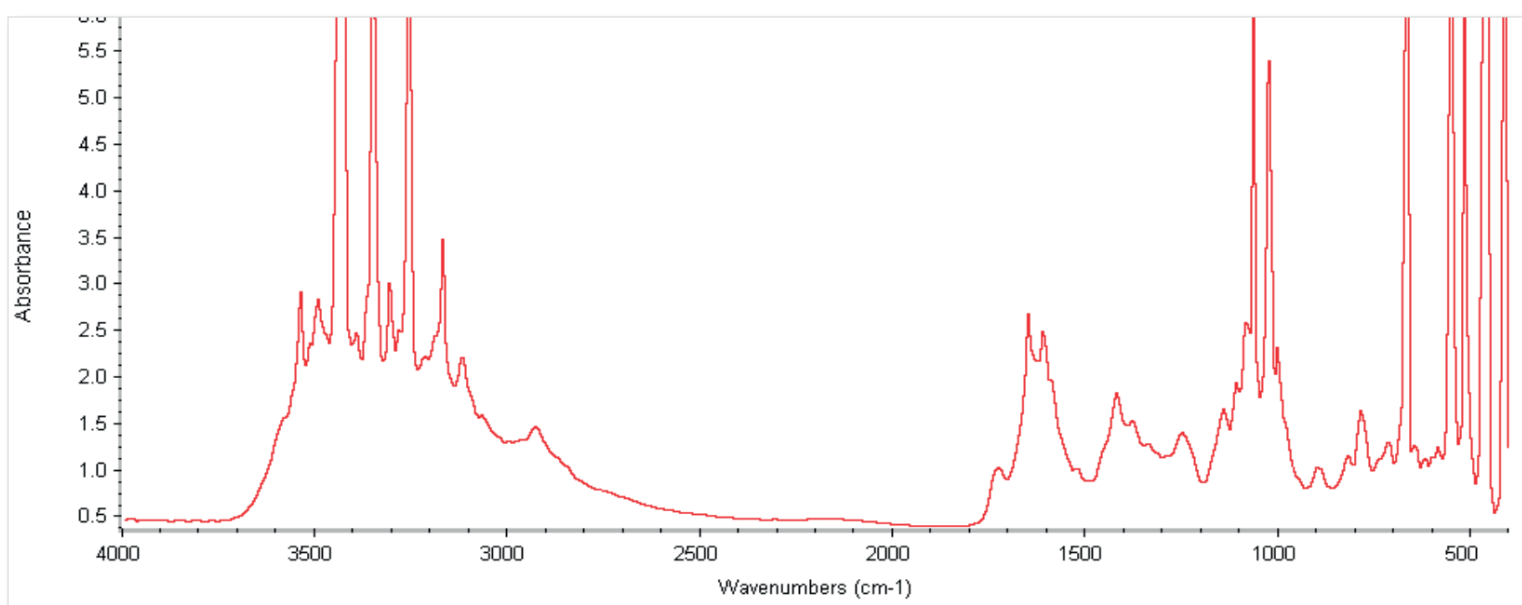

Una vez caracterizado el mucílago se formuló y caracterizó el inhibidor de incrustaciones en sus tres concentraciones, destacando (en la Tabla 3 ) los resultados obtenidos para el pH, el cual incrementó a medida que aumentaba la concentración del mucílago, pero resultando ácido en los tres casos, condición que favorece la acción antiincrustante ya que en medios ácidos aumenta a la disolución del carbonato de calcio (Espinosa, 2015). Un comportamiento similar al anterior se observó en otros parámetros como el contenido de sólidos totales en los productos, influyendo directamente en la densidad de los mismos, al igual que en la viscosidad, ya que a mayor cantidad de sólidos en los inhibidores creció progresivamente la resistencia al flujo. La misma tendencia directamente proporcional concentración-viscosidad se observó en el análisis realizado por Rendón y Azocar (2016) a un inhibidor de incrustaciones a base de Pectina de parchita deshidratada usando partículas de pectina inferiores a los 420 micrones, sin embargo, los valores de viscosidad resultantes fueron mucho menores que los obtenidos con el mucílago de cayena, teniéndose 9.48; 492 y 1938.75 cPs para las concentraciones de 2,4 y 6 \%m/V de su producto.

Tabla 3. Propiedades físicas de los inhibidores formulados con el mucílago de la hoja de cayena (Hibiscus rosa sinensis)

\begin{tabular}{|c|c|c|c|}
\hline Concentración $(\% \mathrm{~m} / \mathbf{V})$ & $\mathrm{pH}$ & Sólidos $(\mathbf{p p m})$ & Viscosidad $(\mathrm{cPs})$ \\
\hline 2 & 4.17 & 15920 & 97.3 \\
\hline 4 & 4.43 & 31684 & 1072 \\
\hline 6 & 4.46 & 47860 & 3359 \\
\hline
\end{tabular}

En cuanto a las características químicas (Tabla 4), destacó el incremento directamente proporcional de los cloruros y la dureza cálcica con concentración, producto del aporte de iones $\mathrm{Cl}^{-}$y $\mathrm{Ca}^{+2}$ por parte del mucílago empleado como materia prima. 
En el caso de la dureza cálcica los resultados obtenidos fueron considerablemente altos si se comparan con la caracterización del inhibidor a base de Pectina de parchita deshidratada (Rendón y Azocar, 2016), donde a pesar de que la propiedad también aumentó con concentración, los valores fueron mucho más bajos, obteniéndose 0.35 ; 0.5 y 0.7 ppm para las concentraciones de 2 , 4 y $6 \% \mathrm{~m} / \mathrm{V}$, respectivamente. Por su parte, los resultados obtenidos por Rendón y Azocar para los cloruros no presentaron variación con concentración, resultando 10 ppm para las tres muestras de inhibidor.

Tabla 4. Propiedades químicas de los inhibidores formulados con el mucílago de la hoja de cayena (Hibiscus rosa sinensis)

\begin{tabular}{|c|c|c|}
\hline Concentración $(\% \mathrm{~m} / \mathrm{V})$ & Cloruros $(\mathrm{ppm})$ & Dureza cálcica (ppm) \\
\hline 2 & 29.9932 & 640 \\
\hline 4 & 59.9865 & 960 \\
\hline 6 & 99.9775 & 1600 \\
\hline
\end{tabular}

Los valores de las propiedades obtenidas para el inhibidor comercial (Tabla 5) muestran que se trata de una sustancia ácida $(\mathrm{pH}<7)$, con una densidad ligeramente superior a la del agua común (1 mg/l), presentó también una ligera coloración amarilla y facilidad a fluir producto de una baja viscosidad. Químicamente se evidenció la ausencia de calcio en su composición y la presencia de iones $\mathrm{Cl}$. Al tratarse de un producto industrialmente probado, sus propiedades físicas y químicas garantizaron cierto poder antiincrustante, lo que permitió establecerlo como patrón referencial en el análisis estadístico del inhibidor propuesto.

Tabla 5. Propiedades del inhibidor comercial

\begin{tabular}{|c|c|c|c|}
\hline $\mathrm{pH}$ & Viscosidad (cPs) & Dureza (ppm) & Cloruros (ppm) \\
\hline 5.58 & 1.32 & 0 & 3699.16 \\
\hline
\end{tabular}

Una vez realizadas las caracterizaciones, se procedio a evaluar la eficiencia del inhibidor en sus tres concentraciones, las cuales a su vez fueron trabajadas en tres dosis diferentes, tanto en muestras de agua sintética como de producción. Durante la prueba de eficiencia, el inhibidor trabajó en función de mantener el contenido de calcio lo más cercano posible al valor de la solución inicial o blanco frío, pues una disminución en dicho contenido indicaría que el resto del calcio ha reaccionado químicamente, precipitándose y generando las incrustaciones. El mucílago empleado en este estudio, al tratarse de un polisacárido con alto contenido de grupos carboxílicos, presenta un efecto quelante, es decir, posee la capacidad de formar iones complejos con los iones metálicos $\left(\mathrm{Ca}^{+2}, \mathrm{Ba}^{+2}, \mathrm{Mg}^{+2} \ldots\right)$ presentes en una solución, evitando que los mismos precipiten en forma de sales (García, 2010).

En este contexto, se pudo observar que el bioinhibidor propuesto presentó una eficiencia máxima en la muestra de agua sintética de $36.73 \%$ correspondiente al inhibidor de $2 \% \mathrm{~m} / \mathrm{V}$ en una dosis de 500 ppm. Además, se evidenció que a mayor concentración menor fue el carácter inhibitorio, esto debido principalmente a la elevada dureza del producto, el cual a medida que aumentaba su contenido de mucílago añadía más calcio al sistema, pudiendo sobrepasar la capacidad de los agentes quelantes también presentes en el producto y, por ende, haciendo más difícil mantener la totalidad del contenido del elemento metálico en solución (Figura 6). 
En cuanto a los resultados de la evaluación en la muestra de agua de producción, representados en la Figura 7, ocurrió también una disminución de la eficiencia a mayor concentración del inhibidor, producto del aporte cálcico ascendente generado por el mismo, que dificultó la inhibición por interacción directa entre los agentes quelantes y los iones incrustantes presentes en el agua. Sin embargo, los valores de eficiencia resultaron mayores que los obtenidos en el agua sintética con un porcentaje máximo de 83.33 \% de inhibición, también correspondientes al inhibidor de $2 \% \mathrm{~m} / \mathrm{V}$ en una dosis de $500 \mathrm{ppm}$.

Figura 6. Comparación de eficiencias entre el inhibidor de 2, 4 y 6 \%m/V de concentración del mucílago de la hoja de cayena (Hibiscus rosa sinensis) y el producto comercial, en muestra de agua sintética

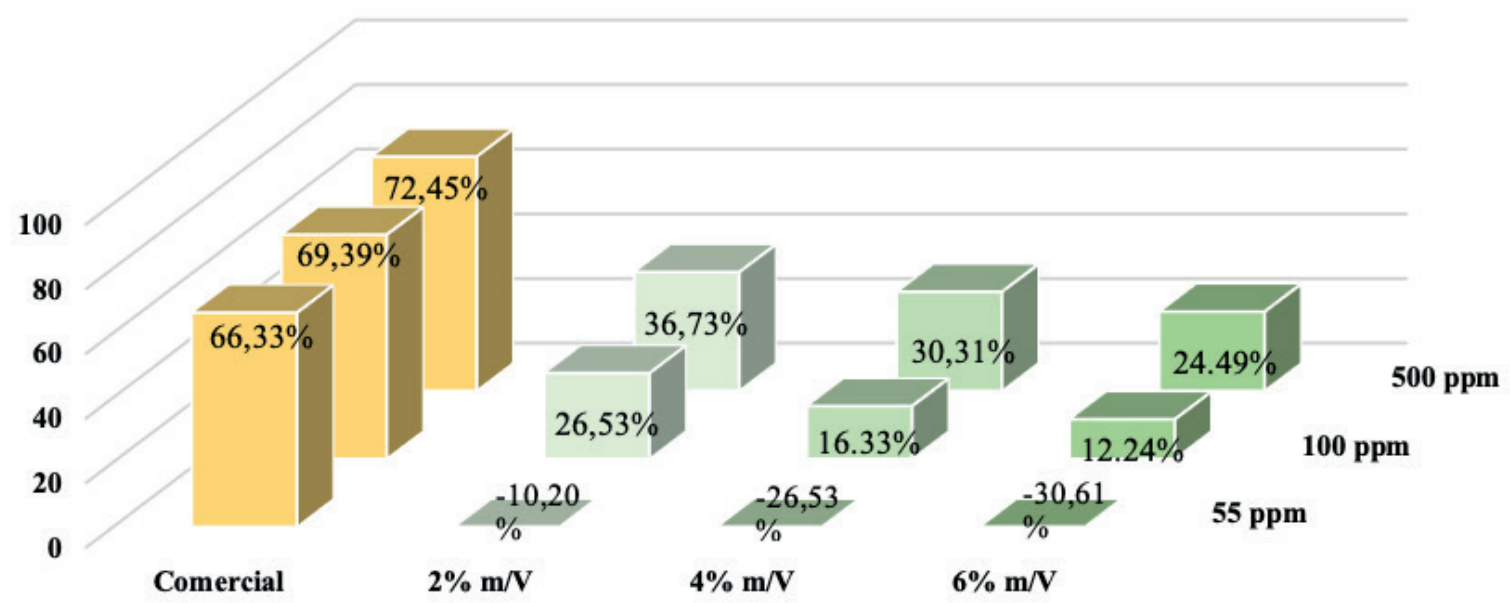

Figura 7. Comparación de eficiencias entre el inhibidor de 2, 4 y 6 \%m/V de concentración del mucílago de la hoja de cayena (Hibiscus rosa sinensis) y el producto comercial, en muestra de agua de producción

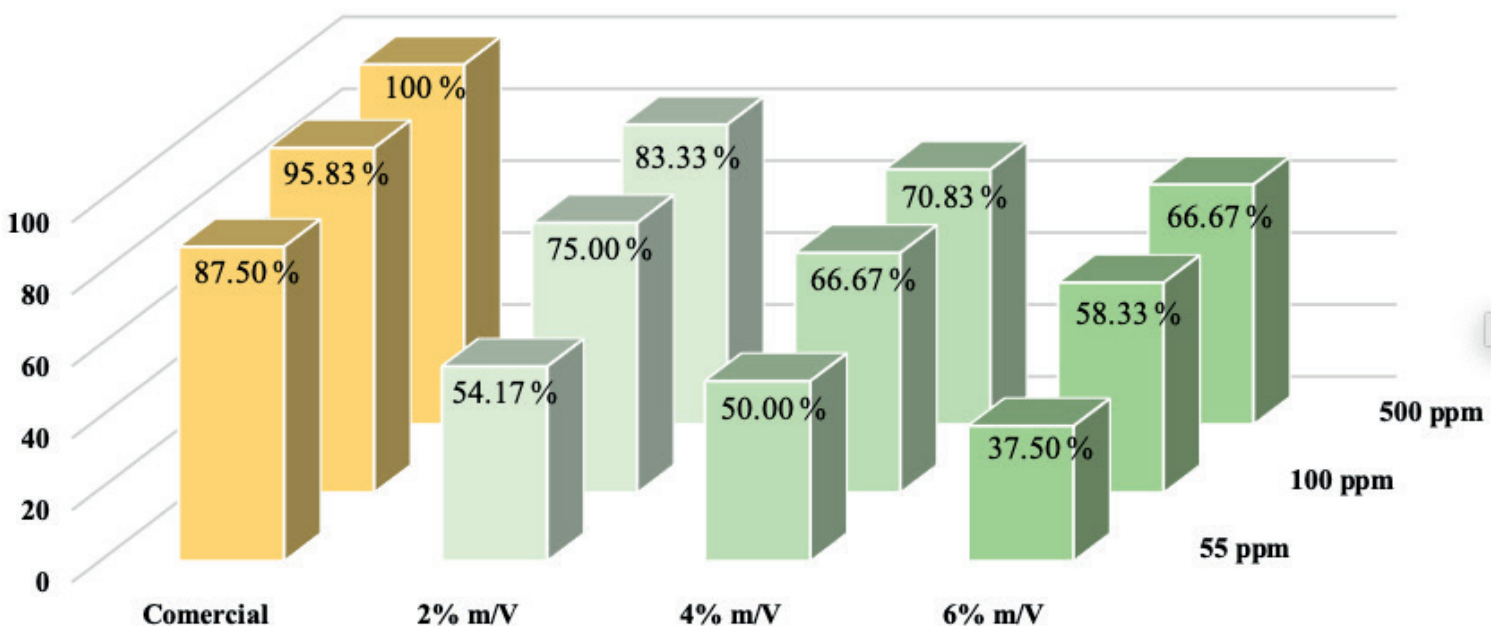


Este contraste, con respecto al comportamiento anterior, pudo deberse al hecho de que las sales empleadas en la formulación de la salmuera, al unirse, crean una reacción química inmediata que generó la formación de un porcentaje de carbonato de calcio $\left(\mathrm{CaCO}_{3}\right)$ antes de que el inhibidor pudiera actuar, generando una disminución inicial del calcio en solución que no puede ser revertida, debido a que se trata de un compuesto químico que previene la precipitación de compuestos minerales, pero no disuelve los depósitos que ya se han formado. Adicionalmente, se evidenció en ambos casos que a medida que se incrementaba la dosis la eficiencia aumentó, dejando ver que más volumen del producto favorece el desempeño inhibitorio.

Es preciso mencionar que aunque el inhibidor de incrustaciones natural a base del mucílago de la hoja de la planta de cayena (Hibiscus rosa sinensis) funcionó en su concentración más baja en ambas muestras de agua, las eficiencias alcanzadas no lograron superar las obtenidas con el bioinhibidor de incrustaciones desarrollado y evaluado por Azócar y Rendón (2016), cuya materia prima fue la pectina deshidratada a base de parchita (Passiflora edulis) y con la cual obtuvieron una inhibición máxima de 89.17 \% correspondiente al inhibidor de 6 \% m/V de concentración, dosis de 100 ppm y tamaño de partículas menor o igual a 420 micrones, probado en muestras de agua sintética.

Estadísticamente, el análisis de varianza realizado con los resultados obtenidos en las muestras de agua sintética reveló la existencia de una diferencia mínima significativa entre las medias de las eficiencias arrojadas por el bioinhibidor en sus tres concentraciones y las del patrón referencial comercial, debido a que el valor $\mathrm{P}$ de la razón $\mathrm{F}$ fue menor que 0.05 en todos los casos, siendo indicativo de que el químico comercial fue capaz de mantener mayor contenido de calcio en solución que el producto natural en las muestra de agua estudiada (Tabla 6).

Tabla 6. Análisis de varianza para eficiencias de los inhibidores en muestra de agua sintética

\begin{tabular}{|l|c|c|c|c|}
\hline \multicolumn{5}{|c|}{ Eficiencias } \\
\hline \multicolumn{1}{|c|}{ Parámetro } & Comercial & $2 \% \mathrm{~m} / \mathrm{V}$ & $\mathbf{4} \% \mathrm{~m} / \mathbf{V}$ & $\mathbf{6} \% \mathrm{~m} / \mathbf{V}$ \\
\hline Valor-P & - & 0.0225 & 0.0222 & 0.0160 \\
\hline Media & 69.4000 & 17.7467 & 6.8033 & 2.0400 \\
\hline Diferencia & - & -51.6533 & -62.5967 & -67.3600 \\
\hline Límites & - & 39.7013 & 47.9148 & 46.6298 \\
\hline Sig & - & $*$ & $*$ & $*$ \\
\hline
\end{tabular}

* Indica una diferencia estadísticamente significativa.

Esto a su vez se ve plasmado en la Figura 8, donde se observa que en el caso del inhibidor a base de mucílago, la tendencia desplegada para cada una de las concentraciones fue muy similar entre ellas, evidenciándose en los tres casos una nula inhibición para la dosis más baja (50 ppm) e inferior al $40 \%$ en las dos dosis restantes (100 y 500 ppm); mientras que el inhibidor comercial mostró una tendencia de pendiente positiva con resultados superiores al $70 \%$ de eficiencia en cada una de las repeticiones. 
Figura 8. Comparación del comportamiento del inhibidor formulado con mucílago de la hoja de cayena (Hibiscus rosa sinensis) a 2, 4 y 6 \% m/V con respecto al inhibidor comercial en muestra de agua sintética

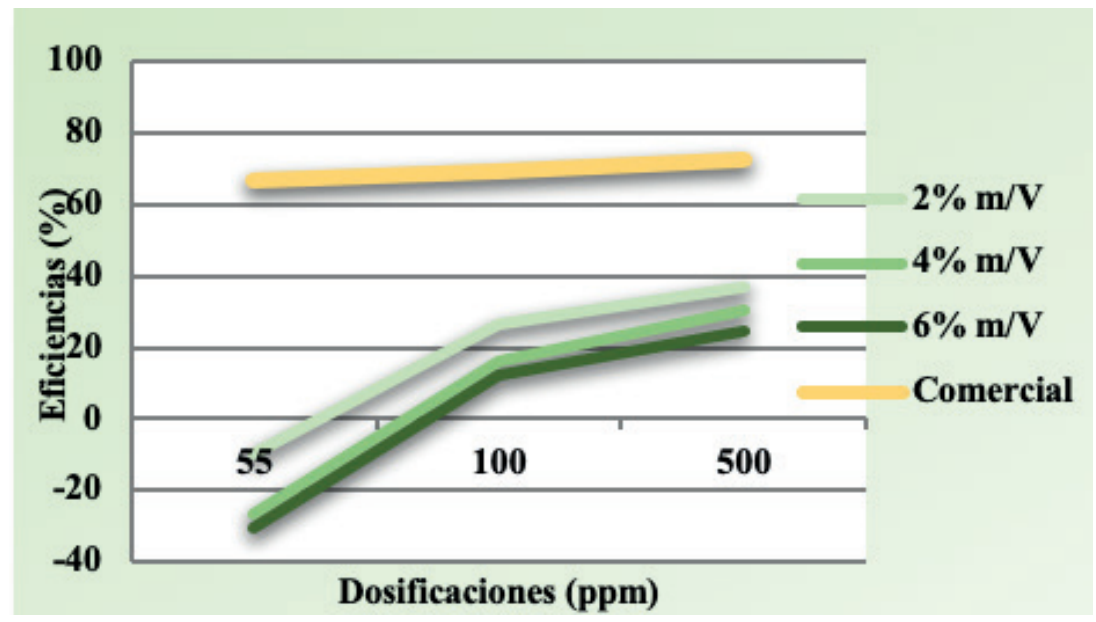

Con respecto a los resultados obtenidos en la muestra de agua de producción, el comportamiento del inhibidor natural en las concentraciones de 2, 4 y 6 \%m/V logró una reproducción bastante similar del patrón ascendente obtenido con el inhibidor comercial en las tres dosificaciones (Figura 9), lo que se traduce en un aumento progresivo de la capacidad de dichos químicos de mantener las sales en solución, contrarrestando el carácter incrustante del agua.

Figura 9. Comparación del comportamiento del inhibidor formulado con mucílago de la hoja de cayena (Hibiscus rosa sinensis) a 2, 4 y $6 \% \mathrm{~m} / \mathrm{V}$, con respecto al inhibidor comercial en muestra de agua de producción

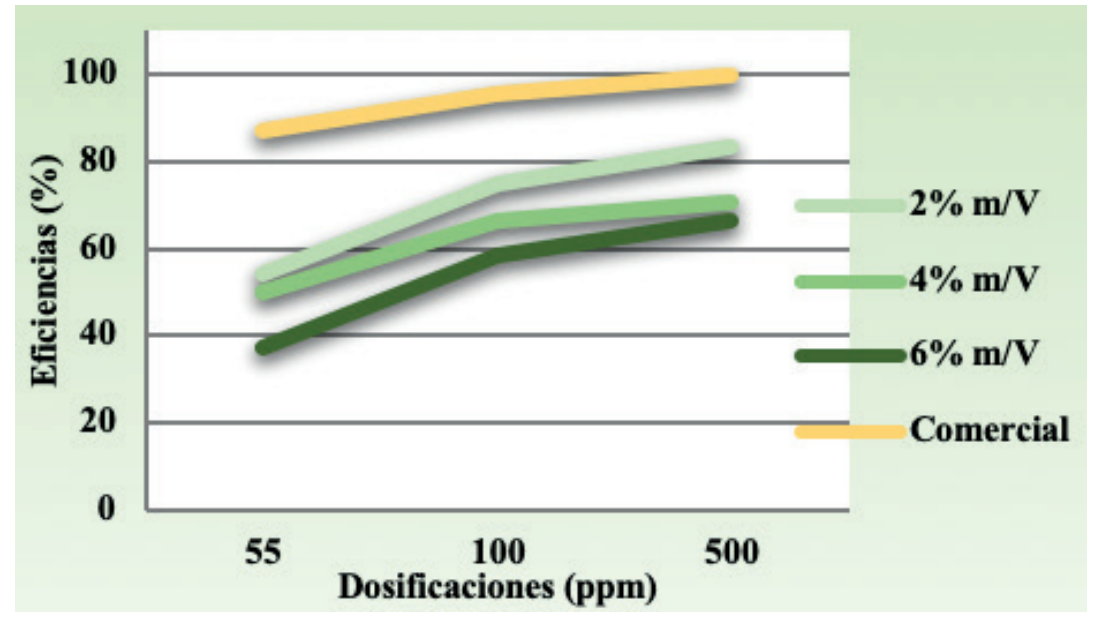

Sin embargo, en el caso de los inhibidores de 4 y 6 \% m/V el estudio mostró la existencia de una diferencia estadística marcada, determinada a través del valor $\mathrm{P}$ de la prueba $\mathrm{F}$ el cual resultó menor que 0.05, siendo esto indicativo de que, aunque los inhibidores funcionaron no alcanzaron un desempeño igual de satisfactorio que el producto químico probado industrialmente. No obstante, para el inhibidor de $2 \% \mathrm{~m} / \mathrm{V}$ se determinó que no existe una diferencia estadísticamente significativa entre la media de eficiencias del mismo y la del inhibidor comercial 
(Tabla 7), precisando que el bioinhibidor en su concentración más baja fue capaz de reproducir la conducta patrón en el mantenimiento eficiente de sales de calcio en suspensión.

Tabla 7. Análisis de varianza para eficiencias de los inhibidores en muestra de agua de producción.

\begin{tabular}{|l|l|l|l|l|}
\hline \multicolumn{5}{|c|}{ Eficiencias } \\
\hline \multicolumn{1}{|c|}{ Parámetro } & \multicolumn{1}{|c|}{ Comercial } & \multicolumn{1}{c|}{$\mathbf{2} \% \mathrm{~m} / \mathbf{N}$} & \multicolumn{1}{c|}{$\mathbf{4} \% \mathrm{~m} / \mathbf{V}$} & \multicolumn{1}{c|}{$\mathbf{m} / \mathbf{V}$} \\
\hline Valor-P & - & 0.0671 & 0.0124 & 0.0131 \\
\hline Media & 94.3867 & 70,8333 & 62.5000 & 54.1667 \\
\hline Diferencia & - & -23.5533 & -31.8867 & -40.2200 \\
\hline Límites & - & 26.2073 & 20.4786 & 26.2135 \\
\hline Sig & - & & $*$ & $*$ \\
\hline
\end{tabular}

* Indica una diferencia estadísticamente significativa.

Los resultados obtenidos se asemejan a los conseguidos por Fuenmayor y Peña (2016) en el análisis estadístico realizado en su estudio a un inhibidor de incrustaciones a base de pectina natural de parchita en muestras de agua de sintética, el cual indicó que el producto con pH de 3,73 no desplegó una tendencia óptima, estandarizándose a las dos primeras concentraciones $(20 \% \mathrm{~m} / \mathrm{V}$ y $30 \% \mathrm{~m} / \mathrm{V})$, y siendo incapaz de reproducir la conducta patrón en el tercer tratamiento $(40 \% \mathrm{~m} / \mathrm{V})$ al momento de aplicar la última dosificación (100 ppm), en contraste con el EDTA y el $\mathrm{HCl}$ usados como agentes referenciales que manifestaron un comportamiento ascendente en respuesta al incremento de sus presencias en los sistemas, como se observa en la figura 10. A su vez se ve reflejado en el Análisis de Varianza (Tablas 8 y 9) que el inhibidor no logró emular ninguna de las conductas, presentando diferencias estadísticas en sus tres dosificaciones, siendo la media más próxima a alguno de los patrones (EDTA), la exhibida por la pectina al $40 \% \mathrm{~m} / \mathrm{V}$.

Figura 10. Comparación del comportamiento del inhibidor de incrustaciones a base de pectina natural de parchita formulado a 20, 30 y $40 \% \mathrm{~m} / \mathrm{V}$ con respecto a HCl y EDTA

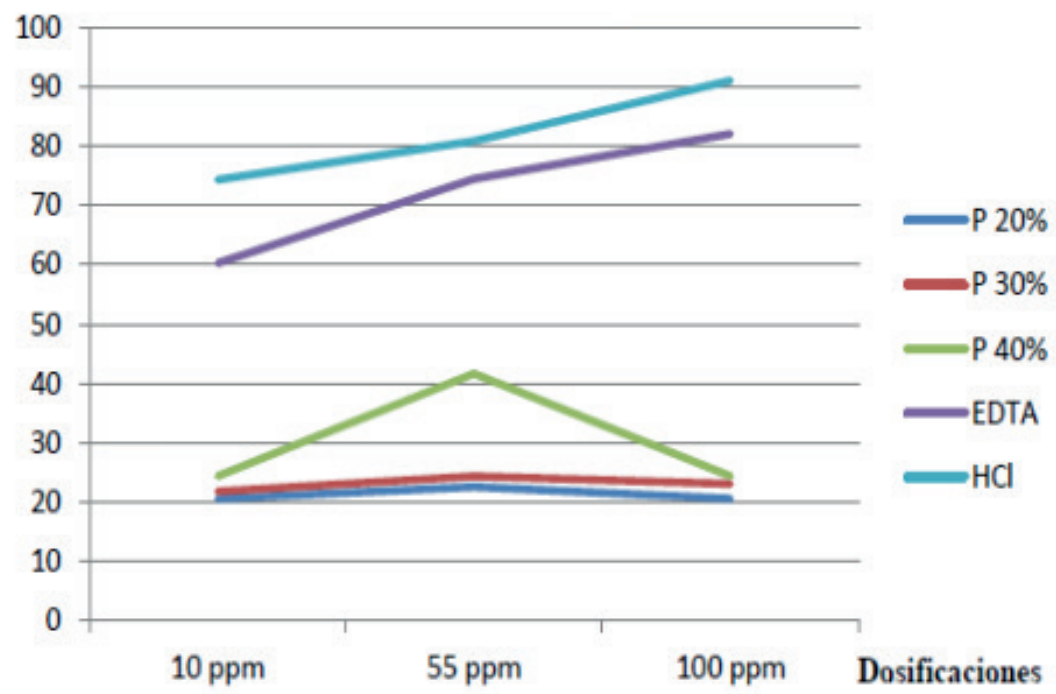

(Tomado: Fuenmayor y Peña, 2016) 
Tabla 8. Análisis de varianza para eficiencias de los inhibidores de pectina (pH 3.73) y EDTA.

\begin{tabular}{|l|c|c|c|c|}
\hline \multirow{2}{*}{ Parámetro } & \multicolumn{4}{|c|}{ Eficiencias (\%) } \\
\cline { 2 - 5 } & EDTA & Pectina (20 \%) & Pectina (30 \%) & Pectina (40 \%) \\
\hline Valor-P & - & 0.0014 & 0,0016 & 0.0081 \\
\hline Media & 72.2633 & 21.1933 & 23,0733 & 30.13 \\
\hline Diferencia & - & -51.07 & $-49,19$ & -42.1333 \\
\hline$+/$ - Límites & - & 17.8348 & 17.8519 & 23.8982 \\
\hline Sig. & - & $*$ & $*$ & $*$ \\
\hline
\end{tabular}

(Tomado de: Fuenmayor y Peña, 2016)

Tabla 9. Análisis de varianza para eficiencias de los inhibidores de pectina $(\mathrm{pH} 3,73)$ y $\mathrm{HCl}$

\begin{tabular}{|l|c|c|c|c|}
\hline \multirow{2}{*}{ Parámetro } & \multicolumn{4}{|c|}{ Eficiencias (\%) } \\
\cline { 2 - 5 } & $\mathbf{H C l}$ & Pectina (20 \%) & Pectina (30 \%) & Pectina (40 \%) \\
\hline Valor-P & - & 0.0002 & 0.0003 & 0.0023 \\
\hline Media & 82.0733 & 21.1933 & 23.0733 & 30.13 \\
\hline Diferencia & - & -60.88 & -59.0 & -51.9433 \\
\hline +/- Límites & - & 13.6049 & 13.6273 & 20.9317 \\
\hline Sig. & - & $*$ & $*$ & $*$ \\
\hline
\end{tabular}

(Tomado de: Fuenmayor y Peña, 2016)

Si se compara el producto desarrollado con el realizado por Chauhan, et al (2012), se aprecia una notable diferencia, toda vez que en este último se alcanzaron eficiencias entre 97,7\% y $100 \%$ para dos tipos de pectinas sintetizadas; diferencias que pueden ser atribuidas principalmente a que los polímeros de ese estudio fueron sintetizados precisamente para mejorar su rendimiento y en el caso del producto a base de Hibiscus rosa sinensis, se empleó el polímero en forma natural, sin someterlo a ningún cambio químico. Adicionalmente, al contrastar el bioinhibidor en estudio con los otros dos prospectos (inhibidor a base de pectina de Passiflora edulis en condición gel y deshidratada) realizados por Fuenmayor y Peña (2016) y Rendón y Azocar (2016) respectivamente, se evidencia un mejor comportamiento que el de la pectina en gel, considerando que en el caso propio se trabajó a concentraciones más bajas, pero sin llegar a ser tan prospectivo como en el caso de la pectina deshidratada (producto formulado con tamaños de partículas pequeñas). Razón por la cual se hace imperativo continuar los estudios al mucílago proveniente de la hoja de Hibiscus rosa sinensis a fin de ajustar un producto inhibidor que esté acorde tanto a los preceptos de la química verde como a los de un anti incrustante con características de alta eficiencia que no genere tantos agregados sólidos al sistema en estudio.

\section{Conclusiones y recomendaciones}

El proceso de extracción permitió determinar que el rendimiento del mucílago de cayena disminuyó considerablemente al deshidratarse, a causa del elevado porcentaje de humedad que posee, además de que las partículas más grandes obtenidas en el proceso de trituración resultaron difíciles de disolver lo cual generó el aporte de cierto porcentaje de sólidos a los inhibidores que interfirieron en su desempeño. Adicionalmente, dicho desempeño del inhibidor de 
incrustaciones a base del mucílago proveniente de la hoja de cayena resultó más favorable en muestras de agua de producción que en muestras de agua sintética con tendencia a una precipitación temprana de $\mathrm{CaCO}_{3^{\prime}}$ obteniéndose mejores resultados al emplear dosis más altas y describiendo un comportamiento inversamente proporcional concentración-eficiencia debido al aporte de calcio por parte de la materia prima que, al incrementarse, dificulta el mecanismo de acción quelante del producto. Es por ello que, como recomendación, resulta conveniente evaluar el uso del mucílago en condición húmeda o emplear únicamente las partículas de menor tamaño en la formulación del producto.

\section{Bibliografía}

American Petroleum Institute (2017). API RP 13B-1 Recommended Practice for Field Testing Water-based

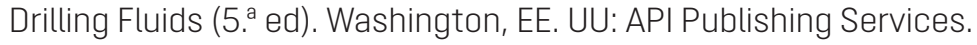

ASTM International (2001). ASTM C136-01, Standard Test Method for Sieve Analysis of Fine and Coarse Aggregates. ASTM International, West Conshohocken, PA, www.astm.org. doi: 10.1520/C0136-01

ASTM International (2008). ASTM E1148-02 Standard Test Method for Measurements of Aqueous Solubility (Withdrawn 2013). ASTM International, West Conshohocken, PA, www.astm.org. doi: 10.1520/ E1148-02R08.

ASTM International (2013). ASTM E1252-98(2013) e1, Standard Practice for General Techniques for Obtaining Infrared Spectra for Qualitative Analysis. ASTM International, West Conshohocken, PA, www.astm.org. doi: 10.1520/E1252-98R13E01

ASTM International (2014). ASTM D854 - Standard Test methods for specific gravity of soil solids by water Pycnometer. ASTM International, West Conshohocken, PA, www.astm.org. doi: 10.1520 / D0854-14.

ASTM International (2016). ASTM E168-16, Standard Practices for General Techniques of Infrared Quantitative Analysis. ASTM International, West Conshohocken, PA, www.astm.org. doi: 10.1520/ E0168-16.

ASTM International (2018). D2196-18e1 Standard Test Methods for Rheological Properties of Non-Newtonian Materials by Rotational Viscometer. ASTM International, West Conshohocken, PA, www. astm.org. doi: 10.1520/D2196-18E01

Canul, M. y Cortes, M. (2015). Disolución de incrustaciones inorgánicas mediante la aplicación de agente quelantes. Método correctivo. Ingeniería petrolera, 55(3), 170-182.

Castillo, L. (2016). Importancia de la caracterización de aguas producidas en la industria petrolera. Geominas, Venezuela, Vol. 44, No. 71, pp 171-175. ISSN 0016-7975.

Chauhan, K., Kumar, R., Kumar, M., Sharma, P. y Chauhan, G. (2012). Modified pectin-based polymers as green antiscalants for calcium sulfate scale inhibition. Desalination, 356, 47-55.

Comisión Venezolana de Normas Internacionales (1986). COVENIN 2342-86 Agua potable. Determinación del residuo filtrable total secado a $180^{\circ} \mathrm{C}$ (Sólidos disueltos). Caracas, Venezuela: Fondonorma.

Comisión Venezolana de Normas Internacionales (1987). COVENIN 2461-87 Aguas naturales, industriales y residuales. Determinación de sólidos. Caracas, Venezuela: Fondonorma.

Comisión Venezolana de Normas Internacionales (1986). COVENIN 2462-87 Aguas naturales, industriales y residuales. Determinación del pH. Caracas, Venezuela: Fondonorma.

Comisión Venezolana de Normas Internacionales (1991). COVENIN 2771-91 Aguas naturales, industriales y residuales. Determinación de dureza. Caracas, Venezuela: Fondonorma.

Comisión Venezolana de Normas Internacionales (1995). COVENIN 3141-95 Aguas naturales, industriales y residuales. Determinación de cloruros. Caracas, Venezuela: Fondonorma.

Espinosa, J. (2015). Combate exitoso de incrustaciones minerales en el sistema de ductos de los Activos de Producción Samaria-Luna y Macuspana-Muspac, (campos petroleros Tizón, Cráter y Coste- 
ro). Metodología para identificar, prevenir y combatir la formación de incrustaciones en instalaciones superficiales de producción. Ingeniería petrolera, México, 55(11).

Fevang, S. (2017). Synthesizing and Testing for New Biodegradable Scale Inhibitors. (Tesis de Maestría). Universidad de Stavanger. Noruega.

Fink, J. (2012). Petroleum Engineer's Guide to Oilfield Chemicals and Fluids. Nueva York, EE.UU: Gulf Professional Publishing.

Fuenmayor, A., y Peña, R. (2016). Evaluación de una pectina natural a base de parchita (Passiflora edulis) como inhibidor de incrustaciones en muestras de agua sintética. Tesis de grado, Universidad de Oriente. Monagas, Venezuela.

García, J. (2010). Desarrollo del Ciclo Tecnológico de un Inhibidor de Incrustaciones Verde para la Industria de los Hidrocarburos: INTAV. Visión tecnológica. Recuperado de: https://www.researchgate. net/journal/1315-0855_Vision_Tecnologica.

Guo, X., Qiu, F., Dong, K., Zhou, X., Qi, J., Zhou, Y., y Yang, D. (2012). Preparation, characterization and scale performance of scale inhibitor copolymer modification with chitosan. Journal of industrial and Engineering Chemistry, 18, 2177-2183.

Jenkins, D. y Snoeyink, V. (2003). Química del Agua. Ciudad de México, México: LIMUSA.

Kelland, M. (2014). Production Chemicals for the Oil and Gas Industry (2. ${ }^{\text {a }}$ ed.). Florida, Estados Unidos: Taylor and Francis Group.

Luzuriaga, D. (2012). Extracción y aprovechamiento del mucílago de Cacao (Theobroma cacao) como materia prima en la elaboración de vino. Universidad Tecnológica Equinoccial. Quito, Ecuador.

Malavé, D. (2016). Evaluación del producto biodegradable (Abelmoschus esculentus) para prevenir la precipitación del carbonato de calcio presente en aguas del Campo El Furrial, Estado Monagas, Universidad de Oriente, Monagas, Venezuela.

Mendoza, B., Gómez, E., Hernández, E., Rodríguez, A., y Chavarría, N. (2014). Elaboración y caracterización de películas biodegradables a partir de mucílago de nopal-caseinato de sodio y mucílago de nopal-pectina. [Artículo en línea]. Recuperado de: https://www.ecorfan.org/handbooks/Ciencia\%20Agropecuarias\%20TII/Articulo_14.pdf

Monrroy, M., García, E., Ríos, K., y García, J. (2017). Extracción y caracterización fisicoquímica del mucílago de la Opuntia cochenillifera (L.) Miller. Journal of Chemistry [Revista en línea]. Recuperado de: https://www.hindawi.com

NACE International (2007). NACE Standard TM0374-2007 Standard Test Method: Laboratory Screening Tests to Determine the Ability of Scale Inhibitors to Prevent the Precipitation of Calcium Sulfate and Calcium Carbonate from Solution (for Oil and Gas Production Systems)..

Rendón, K. y Azócar, E. (2016). Evaluación de la aplicabilidad de pectina deshidratada a base de parchita (Passiflora edulis) como inhibidor de incrustaciones minerales. Tesis de grado, Universidad de Oriente. Monagas, Venezuela.

Selene, M., Jiménez, M. (2014). Determinación de coeficientes convectivos de transferencia de calor natural y forzada para aletas radiales en agua y agua de mar. Puebla, México.

Viloria, A., Castillo, L., García, J. A., y Biomorgi, J., (2010). Aloe derived scale inhibitor. US Patent 7645 722, assigned to Intevep, S.A., Caracas, VE, 12 enero de 2010. http://www.freepatents online. com/7645722.html. 